Modelling, Analysis and Simulation

Modelling, Analysis and Simulation
MAS Rational positive systems for reaction networks

J.H. van Schuppen

RePORt MAS-E0304 APRIL 30, 2003 
CWI is the National Research Institute for Mathematics and Computer Science. It is sponsored by the Netherlands Organization for Scientific Research (NWO).

$\mathrm{CWI}$ is a founding member of ERCIM, the European Research Consortium for Informatics and Mathematics.

CWI's research has a theme-oriented structure and is grouped into four clusters. Listed below are the names of the clusters and in parentheses their acronyms.

Probability, Networks and Algorithms (PNA)

Software Engineering (SEN)

Modelling, Analysis and Simulation (MAS)

Information Systems (INS)

Copyright (C) 2003, Stichting Centrum voor Wiskunde en Informatica

P.O. Box 94079, 1090 GB Amsterdam (NL)

Kruislaan 413, 1098 SJ Amsterdam (NL)

Telephone +31205929333

Telefax +31205924199

ISSN 1386-3711 


\section{Rational Positive Systems for Reaction Networks}

Mathematics Subject Classification (2000):

93C10, 93B15, 93B17.

Key words and Phrases:

Biochemical reaction networks, rational positive systems, realization, control.

Note:

This work was carried out as part of project

MAS 2.3 RESI and LIFESYSTEMS.

The paper has appeared in:

Luca Benvenuti, Alberto De Santis, Lorenzo Farina (Eds.), Proceedings of the First Multidisciplinary International

Symposium Positive Systems: Theory and Applications

(POSTA.2003) (held in Rome, Italy, 28-30 August 2003),

Lecture Notes in Control and Information Sciences, Volume 294,

Springer, Berlin, 2003, 3-4. 


\title{
Rational Positive Systems for Reaction Networks
}

\author{
Jan H. van Schuppen \\ CWI, P.O. Box 94079, 1090 GB Amsterdam, The Netherlands, \\ J.H.van. Schuppen@cwi.nl
}

\section{Extended abstract}

The purpose of the lecture associated with this paper is to present problems, concepts, and theorems of control and system theory for a subclass of the rational positive systems of which examples have been published as models of biochemical cell reaction networks.

The recent advances in knowledge for the genome of plants, animals, and humans now lead to increased interest in cell biology. Knowledge is needed on how a cell as a functional unit operates biochemically and how the reaction network is influenced by the genome via the enzymes. In principle it is possible to model the complete biochemical reaction network of a cell though this program has so far been carried out only for small compartments of such networks.

Mathematical analysis for such reaction networks then leads to a system of ordinary differential equations or of partial differential equations. Often the ordinary differential equations are of polynomial or of rational form. The number of reactions in a cell can be as high as 15.000 (about half the number of estimated genomes) and the number of chemical compounds as high as 20.000. A detailed mathematical analysis of a mathematical model of the complete cell reaction network may therefore not be possible in the short run. Hence there is an interest to develop procedures to obtain from high-order mathematical models approximations in the form of low-order mathematical models. The formulation of approximate models requires understanding of the dynamics of the system, in particular of its algebraic and graph-theoretic structure and of its rate functions. It is the aim of the author to contribute to this research effort.

In this lecture attention is restricted to mathematical models for biochemical cell reaction networks in the form of rational positive systems. These systems are called positive because the state vector represents masses or concentrations of chemical compounds and the external input vectors represent inputs into the network of externally available chemical compounds and of enzymes produced by the nucleus of the cell. The dynamics of the system is often modelled as a polynomial map but in this lecture attention it is restricted to rational maps (each component equals a quotient 
of two polynomials). Such a dynamics arises for example in the model of MichealisMenten kinetics due to a singular perturbation of a bilinear system. The mathematical model of the glycolysis of Trypanosoma brucei is phrazed almost entirely in terms of a rational positive system and this model is regarded as realistic, see [2]. A book on biochemical reaction networks is that of R. Heinrich and S. Schuster, see [1].

The subclass of rational positive systems considered in this lecture is specific due to the conditions imposed by the modeling of biochemical cell reaction networks. It is precisely because of these physically determined conditions that the subclass merits further study. The properties of such systems differ to a minor extent from those of polynomial systems considered. The graph-theoretic and the algebraic structure of rational positive systems make the analysis interesting. A book on mathematical control and system theory is [3] and a paper on polynomial positive systems is [4].

The main topics of the lecture are:

- The mathematical framework of rational positive systems for biochemical reaction networks.

- The system theoretic results on the interconnection and decomposition of rational positive systems, on the realization problem, and the dissipation and conservation properties.

- The formulation of control problems for biochemical reaction networks and preliminary concepts and results for these problems.

\section{Acknowledgements}

The author acknowledges the stimulation and advice provided by Hans V. Westerhoff and Barbara M. Bakker both of the Department of Cell Physiology, of the Faculty of Earth and Life Sciences, of the Vrije Universiteit in Amsterdam. He also acknowledges discussions on the topic of this lecture with Mr. Siddhartha Jha during an internship of the latter at CWI in the Summer of 2001. He thanks Dorina Jibetean for symbolic calculations for the example. He also thanks several unnamed researchers for comments on drafts of the paper.

\section{References}

1. R. Heinrich and S. Schuster. The regulation of cellular systems. Chapman and Hall, New York, 1996.

2. Sandre Helfert, Antonio M. Estévez, Barbara Bakker, Paul Michels, and Christine Clayton. Roles of triosephosphate isomerase and aerobic metabolism in trypanosoma brucei. Biochem. J., 357:117-125, 2001.

3. E.D. Sontag. Mathematical control theory: Deterministic finite dimensional systems (2nd. Ed.). Number 6 in Graduate Text in Applied Mathematics. Springer, New York, 1998.

4. E.D. Sontag. Structure and stability of certain chemical networks and applications to the kinetic proofreading model of T-cell receptor signal transduction. IEEE Trans. Automatic Control, 46:1028-1047, 2001. 